\title{
Singular sets of separately analytic functions
}

\author{
by ZBigniew BŁOCKI (Kraków)
}

\begin{abstract}
We complete the characterization of singular sets of separately analytic functions. In the case of functions of two variables this was earlier done by J. Saint Raymond and J. Siciak.
\end{abstract}

1. Introduction. If $\Omega$ is an open subset of $\mathbb{R}^{n_{1}} \times \ldots \times \mathbb{R}^{n_{s}}$, then we say that a function $f: \Omega \rightarrow \mathbb{C}$ is p-separately analytic $(1 \leq p<s)$ if for every $x^{0}=\left(x_{1}^{0}, \ldots, x_{s}^{0}\right) \in \Omega$ and for every sequence $1 \leq i_{1}<\ldots<i_{p} \leq s$ the function

$$
\left(x_{i_{1}}, \ldots, x_{i_{p}}\right) \rightarrow f\left(x_{1}^{0}, \ldots, x_{i_{1}}, \ldots, x_{i_{p}}, \ldots, x_{s}^{0}\right)
$$

is analytic in a neighbourhood of $\left(x_{i_{1}}^{0}, \ldots, x_{i_{p}}^{0}\right)$. For a $p$-separately analytic function $f$ in $\Omega$ let

$$
\mathrm{A}(f):=\{x \in \Omega: f \text { is analytic in a neighbourhood of } x\}
$$

denote its set of analyticity, and $\mathrm{S}(f):=\Omega \backslash \mathrm{A}(f)$ its singular set.

If $X$ and $Y$ are any sets, $S \subset X \times Y$ and $\left(x^{0}, y^{0}\right) \in X \times Y$, then we define $S\left(x^{0}, \cdot\right):=\left\{y \in Y:\left(x^{0}, y\right) \in S\right\}, S\left(\cdot, y^{0}\right):=\left\{x \in X:\left(x, y^{0}\right) \in S\right\}$.

The following theorems characterize singular sets of separately analytic functions.

THEOREM A. If $f$ is p-separately analytic in $\Omega$, then for every sequence $1 \leq j_{1}<\ldots<j_{q} \leq s$, where $q:=s-p$, the projection of $\mathrm{S}(f)$ on $\mathbb{R}^{n_{j_{1}} \times}$ $\ldots \times \mathbb{R}^{n_{j_{q}}}$ is pluripolar $\left(\right.$ in $\left.\mathbb{C}^{n_{j_{1}}} \times \ldots \times \mathbb{C}^{n_{j_{q}}}\right)$.

THEOREM B. Let $S$ be a closed subset of $\Omega$ such that for every sequence $1 \leq j_{1}<\ldots<j_{q} \leq s$, where $q:=s-p$, the projection of $S$ on $\mathbb{R}^{n_{j_{1}}} \times \ldots \times$ $\mathbb{R}^{n_{j_{q}}}$ is pluripolar. Then there exists a p-separately analytic function $f$ in $\Omega$ such that $S=\mathrm{S}(f)$.

THEOREM C. Let $f$ be $p$-separately analytic in $\Omega$. If $1 \leq k<s$, then for quasi-almost all $x \in \mathbb{R}^{n_{1}} \times \ldots \times \mathbb{R}^{n_{k}}$ (that is, for $x \in \mathbb{R}^{n_{1}} \times \ldots \times \mathbb{R}^{n_{k}} \backslash P$, 
where $P$ is pluripolar $), \mathrm{S}(f(x, \cdot))=\mathrm{S}(f)(x, \cdot)$.

Theorems A and $\mathrm{B}$ in case $s=2, p=n_{1}=n_{2}=1$ were proved by Saint Raymond [2]. This result was generalized by Siciak [5], who proved Theorem A for $p \geq s / 2$ and Theorem B. The aim of this paper is to give a proof of Theorem C; then, as a trivial consequence, we get Theorem A.

2. Preliminaries. We need the following two theorems:

SiciaK's theorem ([3]; see also [4], Theorem 9.7). For $j=1, \ldots, s$ let $D_{j}=D_{j}^{1} \times \ldots \times D_{j}^{n_{j}}$, where the $D_{j}^{t}$ are open sets in $\mathbb{C}$, symmetric about the $x_{t}$-axis $\left(t=1, \ldots, n_{j}\right)$, and $K_{j}=K_{j}^{1} \times \ldots \times K_{j}^{n_{j}}$, where the $K_{j}^{t}$ are closed intervals in $D_{j}^{t} \cap \mathbb{R}$. Let $f$ be a separately holomorphic function in

$$
X:=\bigcup_{j=1}^{s} K_{1} \times \ldots \times D_{j} \times \ldots \times K_{s}
$$

(that is, for every $\left(x_{1}, \ldots, x_{s}\right) \in K_{1} \times \ldots \times K_{s}$ and for every $j=1, \ldots, s$ the function $f\left(x_{1}, \ldots, x_{j-1}, \cdot, x_{j+1}, \ldots, x_{s}\right)$ is holomorphic in $\left.D_{j}\right)$. Then $f$ can be extended to a holomorphic function in a neighbourhood of $\left.X{ }^{1}\right)$.

BEDFord-TAYLOR THEOREM ON NEGLigible SETs [1]. If $\left\{u_{j}\right\}_{j \in J}$ is a family of plurisubharmonic functions locally bounded from above then the set

$$
\left\{z \in D: u(z):=\sup _{j \in J} u_{j}(z)<u^{*}(z)\right\}
$$

is pluripolar ( $u^{*}$ denotes the upper regularization of $u$ ).

\section{Proofs}

Theorem $\mathrm{C} \Rightarrow$ Theorem $\mathrm{A}$ : We may assume that $\left(j_{1}, \ldots, j_{q}\right)=$ $(1, \ldots, q)$. Then it is enough to take $k=q$ and see that for $x \in \mathbb{R}^{n_{1}} \times \ldots \times$ $\mathbb{R}^{n_{k}}, \mathrm{~S}(f(x, \cdot))=\emptyset$.

Proof of Theorem C. We can write

$$
\begin{aligned}
\mathbb{R}^{n_{1}} \times \ldots \times \mathbb{R}^{n_{s}}= & \left(\mathbb{R}^{n_{1}} \times \ldots \times \mathbb{R}^{n_{p}}\right) \times \ldots \times\left(\mathbb{R}^{n_{a p+1}} \times \ldots \times \mathbb{R}^{n_{k}}\right) \\
& \times\left(\mathbb{R}^{n_{k+1}} \times \ldots \times \mathbb{R}^{n_{k+p}}\right) \times \ldots \times\left(\mathbb{R}^{n_{k+b p+1}} \times \ldots \times \mathbb{R}^{n_{s}}\right),
\end{aligned}
$$

where $a=[k / p], b=[(s-k) / p]$. Then $f$ is separately analytic (that is, 1-separately analytic) with respect to such variables. Therefore it is enough to prove Theorem $\mathrm{C}$ for $p=1$. Let $\left\{X_{\nu} \times Y_{\nu}\right\}_{\nu \in \mathbb{N}}$ be a countable family

$\left({ }^{1}\right)$ In fact we use Siciak's theorem under the additional assumption that $f$ is bounded. In this case the proof is much simpler-it can be deduced from Theorem 2a in [3]. 
of closed intervals in $\left(\mathbb{R}^{n_{1}} \times \ldots \times \mathbb{R}^{n_{k}}\right) \times\left(\mathbb{R}^{n_{k+1}} \times \ldots \times \mathbb{R}^{n_{s}}\right)$ such that $\bigcup_{\nu=1}^{\infty} X_{\nu} \times Y_{\nu}=\Omega$. It is clear that

$$
\begin{aligned}
\left\{x \in \mathbb{R}^{n_{1}} \times \ldots \times \mathbb{R}^{n_{k}}: \mathrm{S}(f(x, \cdot)) \varsubsetneqq \mathrm{S}(f)(x, \cdot)\right\} & \\
& \subset \bigcup_{\nu=1}^{\infty}\left\{x \in X_{\nu}: \mathrm{S}(f(x, \cdot)) \cap Y_{\nu} \varsubsetneqq \mathrm{S}(f)(x, \cdot) \cap Y_{\nu}\right\} .
\end{aligned}
$$

Hence we may assume that $f$ is separately analytic in a closed interval $I_{1} \times \ldots \times I_{s} \subset \mathbb{R}^{n_{1}} \times \ldots \times \mathbb{R}^{n_{s}}$ (that is, analytic in some open neighbourhood of this interval).

To prove Theorem $\mathrm{C}$ we have to show that the set

$$
Z_{f, k}:=\left\{x \in I_{1} \times \ldots \times I_{k}: \mathrm{S}(f(x, \cdot)) \varsubsetneqq \mathrm{S}(f)(x, \cdot)\right\}
$$

is pluripolar.

For $(x, y) \in\left(I_{1} \times \ldots \times I_{k}\right) \times\left(I_{k+1} \times \ldots \times I_{s}\right)$ such that $y \in \mathrm{A}(f(x, \cdot))$ define

$$
Q_{f, k}(x, y):=\sup _{|\alpha| \geq 1}\left|\frac{1}{\alpha !} \frac{\partial^{|\alpha|} f}{\partial y^{\alpha}}(x, y)\right|^{1 /|\alpha|}
$$

(of course $Q_{f, k}(x, y)<\infty$ and $f(x, \cdot)$ is holomorphic in the polydisc $P(y$, $\left.\left.1 / Q_{f, k}(x, y)\right)\right)$.

For $y \in I_{k+1} \times \ldots \times I_{s}$ let

$F_{f, k}(y):=\left\{x \in \mathrm{A}(f)(\cdot, y): Q_{f, k}(\cdot, y)\right.$ is not upper semicontinuous at $\left.x\right\}$.

Theorem $\mathrm{C}$ is proved by induction on $k$. First assume that $k=1$.

$1^{\mathrm{o}}$ The projection of $\mathrm{S}(f)$ on $I_{2} \times \ldots \times I_{s}$ is nowhere dense in $\mathbb{R}^{n_{2}} \times$ $\ldots \times \mathbb{R}^{n_{s}}$, that is, there exists an open, dense subset $U$ of $I_{2} \times \ldots \times I_{s}$ such that $I_{1} \times U \subset \mathrm{A}(f)$. In particular, $\mathrm{A}(f)$ is dense in $I_{1} \times \ldots \times I_{s}$.

Proof (induction on $s$ ). The same proof applies to the case $s=2$ and to the step $s-1 \Rightarrow s$. We have

$$
I_{1}=\left[a_{1}, b_{1}\right] \times \ldots \times\left[a_{n_{1}}, b_{n_{1}}\right] .
$$

Define for $m \in \mathbb{N}$

$$
\begin{aligned}
& I_{1}^{m}:=\left\{z \in \mathbb{C}^{n_{1}}: \max _{1 \leq t \leq s} \operatorname{dist}\left(z_{t},\left[a_{t}, b_{t}\right]\right)<1 / m\right\} \\
& E_{m}:=\left\{y_{1} \in I_{2} \times \ldots \times I_{s}: f\left(\cdot, y_{1}\right) \text { is holomorphic in } I_{1}^{m},\right. \\
&\left.\sup _{z \in I_{1}^{m}}\left|f\left(z, y_{1}\right)\right| \leq m\right\} .
\end{aligned}
$$

We have $E_{m} \subset E_{m+1}, \bigcup_{m=1}^{\infty} E_{m}=I_{2} \times \ldots \times I_{s}$. First we want to show that the set $U_{1}:=\bigcup_{m=1}^{\infty}$ int $E_{m}$ is dense in $I_{2} \times \ldots \times I_{s}$. Let $Y^{\prime}$ be a closed interval in $I_{2} \times \ldots \times I_{s}$, and $\mathcal{H}$ a family of closed intervals which form a countable base of the topology in $Y^{\prime}$. For $x_{1} \in I_{1}$ the set $\mathrm{A}\left(f\left(x_{1}, \cdot\right)\right)$ is 
dense: this is trivial if $s=2$ and follows from the inductive assumption if $s \geq 3$. Therefore, if for $H \in \mathcal{H}$ we set

$$
A_{H}:=\left\{x_{1} \in I_{1}: f\left(x_{1}, \cdot\right) \text { is analytic in } H\right\},
$$

it follows that $\bigcup_{H \in \mathcal{H}} A_{H}=I_{1}$. We claim that there exists $H_{0} \in \mathcal{H}$ such that the set $A_{H_{0}}$ is determining for functions holomorphic in a complex neighbourhood of $I_{1}$. Indeed, suppose not. Then all the sets $A_{H}(H \in \mathcal{H})$ are nowhere dense in $I_{1}$ and by the Baire theorem we get a contradiction. Hence, by Montel's lemma, the sets $E_{m} \cap H_{0}(m \in \mathbb{N})$ are closed, and, again by the Baire theorem, $U_{1} \cap H_{0} \neq \emptyset$. Therefore $U_{1}$ is open and dense in $I_{2} \times \ldots \times I_{s}$. Analogously to $I_{1}^{m}$ and $U_{1}$ we define $I_{j}^{m}$ and $U_{j}(j=2, \ldots, s$, $m \in \mathbb{N})$. Take a closed interval $K_{2} \times \ldots \times K_{s} \subset U_{1}$. Since the $U_{j}$ are dense we can find closed intervals $\widetilde{K}_{1} \subset I_{1}, \widetilde{K}_{j} \subset K_{j}(j=2, \ldots, s)$ and $m \in \mathbb{N}$ such that for $j=1, \ldots, s$

$$
\widetilde{K}_{1} \times \ldots \times \widetilde{K}_{j-1} \times \widetilde{K}_{j+1} \times \ldots \times \widetilde{K}_{s} \subset U_{j},
$$

and $f$ is separately holomorphic and bounded by $m$ in

$$
\bigcup_{j=1}^{s} \widetilde{K}_{1} \times \ldots \times I_{j}^{m} \times \ldots \times \widetilde{K}_{s} .
$$

Hence, by Siciak's theorem, $I_{1} \times \widetilde{K}_{2} \times \ldots \times \widetilde{K}_{s} \subset \mathrm{A}(f)$.

$2^{\circ}$ For $y_{1} \in U$ the set $F_{f, 1}\left(y_{1}\right)$ is pluripolar.

Proof. Since $I_{1} \times\left\{y_{1}\right\} \subset \mathrm{A}(f)$ we see that there exist a complex neighbourhood $D$ of $I_{1}$ and a complex neighbourhood $B$ of $y_{1}$ such that $f$ is holomorphic in $D \times B$. By the Bedford-Taylor theorem

$$
N:=\left\{z \in D: \varphi(z):=\sup _{|\alpha| \geq 1}\left|\frac{1}{\alpha !} \frac{\partial^{|\alpha|} f}{\partial y_{1}^{\alpha}}\left(z, y_{1}\right)\right|^{1 /|\alpha|}<\varphi^{*}(z)\right\}
$$

is pluripolar, and of course $F_{f, 1}\left(y_{1}\right) \subset N$.

$3^{\circ}$ If $V$ is a countable and dense subset of $U$ then $Z_{f, 1} \subset \bigcup_{y_{1} \in V} F_{f, 1}\left(y_{1}\right)$.

Proof. Take $x_{1}^{0} \in Z_{f, 1}$. We can find $y_{1}^{0} \in I_{2} \times \ldots \times I_{s}$ such that $\left(x_{1}^{0}, y_{1}^{0}\right) \in \mathrm{S}(f)$, but $y_{1}^{0} \in \mathrm{A}\left(f\left(x_{1}^{0}, \cdot\right)\right)$. Hence $f\left(x_{1}^{0}, \cdot\right)$ is holomorphic in the polydisc $P\left(y_{1}^{0}, 1 / Q_{f, 1}\left(x_{1}^{0}, y_{1}^{0}\right)\right) \subset \mathbb{C}^{N}$, where $N:=n_{2}+\ldots+n_{s}$. Let $\lambda$ be such that $0<\lambda \leq 1 / 4$ and $(1-\lambda)^{-1-N}<2$ and let $r:=\min \{1$, $\left.1 / Q_{f, 1}\left(x_{1}^{0}, y_{1}^{0}\right)\right\}$. For $y_{1} \in \vartheta:=P\left(y_{1}^{0}, \lambda r\right) \subset \mathbb{C}^{N}$ we have

$$
f\left(x_{1}^{0}, y_{1}\right)=\sum_{\alpha} \frac{1}{\alpha !} \frac{\partial^{|\alpha|} f}{\partial y^{\alpha}}\left(x_{1}^{0}, y_{1}^{0}\right)\left(y_{1}-y_{1}^{0}\right)^{\alpha} .
$$


We deduce that

$$
\begin{aligned}
\left|\frac{1}{\beta !} \frac{\partial^{|\beta|} f}{\partial y_{1}^{\beta}}\left(x_{1}^{0}, y_{1}\right)\right| & \leq Q_{f, 1}\left(x_{1}^{0}, y_{1}^{0}\right)^{|\beta|} \sum_{\alpha} \frac{(\alpha+\beta) !}{\alpha ! \beta !} \lambda^{|\alpha|} \\
& =Q_{f, 1}\left(x_{1}^{0}, y_{1}^{0}\right)^{|\beta|}(1-\lambda)^{-|\beta|-N}
\end{aligned}
$$

hence

$$
Q_{f, 1}\left(x_{1}^{0}, y_{1}\right) \leq(1-\lambda)^{-1-N} Q_{f, 1}\left(x_{1}^{0}, y_{1}^{0}\right)<2 / r .
$$

By $1^{\circ}$ there exists $\widetilde{y}_{1} \in \vartheta \cap V$. It is enough to show that $x_{1}^{0} \in F_{f, 1}\left(\widetilde{y}_{1}\right)$. Assume this is not so, that is, $Q_{f, 1}(\cdot, \widetilde{y})$ is upper semicontinuous at $x_{1}^{0}$. Therefore there exists a closed interval $K$, a neighbourhood of $x_{1}^{0}$ in $I_{1}$ such that for $x_{1} \in K$

$$
Q_{f, 1}\left(x_{1}, \widetilde{y}\right)<2 / r
$$

The function $f\left(x_{1}, \cdot\right)$ is holomorphic in a neighbourhood of $\widetilde{y}_{1}$ (because $\widetilde{y}_{1} \in U$, hence $\left.\left(x_{1}, \widetilde{y}_{1}\right) \in \mathrm{A}(f)\right)$ and so it is holomorphic in the polydisc $P\left(\widetilde{y}_{1}, 1 / Q_{f, 1}\left(x_{1}, \widetilde{y}_{1}\right)\right)$. We have

$$
P\left(\widetilde{y}_{1}, 1 / Q_{f, 1}\left(x_{1}, \widetilde{y}_{1}\right)\right) \supset P\left(\widetilde{y}_{1}, r / 2\right) \supset \vartheta,
$$

hence for $x_{1} \in K, f\left(x_{1}, \cdot\right)$ is holomorphic in $\vartheta$. Moreover, for $y_{1} \in \vartheta$ we have

$$
\left|f\left(x_{1}, y_{1}\right)\right| \leq \sum_{\alpha} Q_{f, 1}\left(x_{1}, y_{1}\right)^{|\alpha|}(\lambda r)^{|\alpha|} \leq \sum_{\alpha} 2^{-|\alpha|}=2^{N}
$$

Let $U_{1}$ and $I_{1}^{m}$ be as in the proof of $1^{\circ}$. Take a closed interval $H \subset \vartheta \cap U_{1}$. We can find $m$ such that $f$ is separately holomorphic (as a function of two variables: $x_{1} \in I_{1}$ and $\left.y_{1} \in I_{2} \times \ldots \times I_{s}\right)$ and bounded by $m$ in $K \times \vartheta \cup I_{1}^{m} \times H$. By Siciak's theorem $\left(x_{1}^{0}, y_{1}^{0}\right) \in \mathrm{A}(f)$, a contradiction.

By $2^{\circ}$ and $3^{\circ}$ we deduce that $Z_{f, 1}$ is pluripolar. Thus we have proved the first inductive step: we have shown that Theorem $\mathrm{C}$ is true for $k=1$ and any $s \geq 2$. Now let $k \geq 2$ and assume that Theorem $\mathrm{C}$ is true for $k-1$ and any $s \geq k$.

$4^{\mathrm{o}}$ The set

$$
W:=\left\{y \in I_{k+1} \times \ldots \times I_{s}: \mathrm{S}(f(\cdot, y))=\mathrm{S}(f)(\cdot, y)\right\}
$$

is dense in $I_{k+1} \times \ldots \times I_{s}$.

Proof. As we have just shown Theorem $\mathrm{C}$ is true for $k=1$. Using this $k$ times for any $k>1$ we see that for quasi-almost all $x_{s} \in I_{s}, \ldots$, for quasi-almost all $x_{k+1} \in I_{k+1}$ we have

$$
\mathrm{S}\left(f\left(\cdot, x_{k+1}, \ldots, x_{s}\right)\right)=\mathrm{S}(f)\left(\cdot, x_{k+1}, \ldots, x_{s}\right) .
$$

In particular, $W$ is dense.

$5^{\circ}$ For $y \in W$ the set $F_{f, k}(y)$ is pluripolar. 
Proof. If $L \Subset \mathrm{A}(f)(\cdot, y)$, then in the same way as in the proof of $2^{\circ}$ we show that $F_{f, k}(y) \cap L$ is pluripolar.

$6^{\circ}$ If $W^{\prime}$ is a countable and dense subset of $W$, then the set

$$
R:=Z_{f, k} \backslash \bigcup_{y \in W^{\prime}}\left(\mathrm{S}(f(\cdot, y)) \cup F_{f, k}(y)\right)
$$

is pluripolar.

Proof. Take any $x^{0} \in R$. By the definition of $Z_{f, k}$ we can find $y^{0} \in$ $I_{k+1} \times \ldots \times I_{s}$ such that $\left(x^{0}, y^{0}\right) \in \mathrm{S}(f)$, but $y^{0} \in \mathrm{A}\left(f\left(x^{0}, \cdot\right)\right)$. Define $g:=f\left(x_{1}^{0}, \ldots, x_{k-1}^{0}, \cdot\right)$. First we want to show that $\left(x_{k}^{0}, y^{0}\right) \in \mathrm{A}(g)$. Assume $\left(x_{k}^{0}, y^{0}\right) \in \mathrm{S}(g)$. We have $y^{0} \in \mathrm{A}\left(g\left(x_{k}^{0}, \cdot\right)\right)$, therefore $x_{k}^{0} \in Z_{g, 1}$. By $3^{\circ}$ we can find $y \in W^{\prime}$ such that $x_{k}^{0} \in F_{g, 1}(y)$, that is, $Q_{g, 1}(\cdot, y)$ is not upper semicontinuous at $x_{k}^{0}$. By the definition of $R$ and $W$ we have

$$
x^{0} \in \mathrm{A}(f(\cdot, y)) \backslash F_{f, k}(y)=\mathrm{A}(f)(\cdot, y) \backslash F_{f, k}(y),
$$

whence $Q_{f, k}(\cdot, y)$ is upper semicontinuous at $x_{k}^{0}$. In particular, $Q_{f, k}\left(x_{1}^{0}, \ldots\right.$ $\left.\ldots, x_{k-1}^{0}, \cdot, y\right)=Q_{g, 1}(\cdot, y)$ is upper semicontinuous at $x^{0}$, a contradiction. Thus $\left(x_{k}^{0}, y^{0}\right) \in \mathrm{A}(g)$, hence

$$
\left(x_{k}^{0}, y^{0}\right) \in \mathrm{S}(f)\left(x_{1}^{0}, \ldots, x_{k-1}^{0}, \cdot\right) \backslash \mathrm{S}\left(f\left(x_{1}^{0}, \ldots, x_{k-1}^{0}, \cdot\right)\right),
$$

and so $\left(x_{1}^{0}, \ldots, x_{k-1}^{0}\right) \in Z_{f, k-1}$. We have shown that the projection of $R$ on $I_{1} \times \ldots \times I_{k-1}$ is contained in $Z_{f, k-1}$, which is, by the inductive assumption, pluripolar. In particular, $R$ is pluripolar.

By the inductive assumption Theorem $\mathrm{C}$ is true for any separately analytic function of $k$ variables, hence for such functions Theorem $\mathrm{A}$ is true as well. In particular, for $y \in I_{k+1} \times \ldots \times I_{s}$ the set $\mathrm{S}(f(\cdot, y))$ is pluripolar. Therefore, by $4^{\circ}, 5^{\circ}$ and $6^{\circ}, Z_{f, k}$ is pluripolar. The proof of Theorem $\mathrm{C}$ is complete.

Acknowledgements. I would like to thank Professor Siciak for calling my attention to the problem, for his help in solving it and precious discussions on this material.

\section{References}

[1] E. Bedford and B. A. Taylor, A new capacity for plurisubharmonic functions, Acta Math. 149 (1982), 1-40.

[2] J. Saint Raymond, Fonctions séparément analytiques, Ann. Inst. Fourier (Grenoble) 40 (1990), 79-101.

[3] J. Siciak, Analyticity and separate analyticity of functions defined on lower dimensional subsets of $\mathbb{C}^{n}$, Zeszyty Nauk. Uniw. Jagielloń. Prace Mat. 13 (1969), 53-70. 
[4] J. Siciak, Separately analytic functions and envelopes of holomorphy of some lower dimensional subsets of $\mathbb{C}^{n}$, Ann. Polon. Math. 22 (1969), 145-171.

[5] —, Singular sets of separately analytic functions, Colloq. Math. 60/61 (1990), 281290.

INSTITUTE OF MATHEMATICS

JAGIELLONIAN UNIVERSITY

REYMONTA 4

30-059 KRAKÓW, POLAND

E-MAIL: UMBLOCKI@PLKRCY11.BITNET

Reçu par la Rédaction le 30.5.1991 